\title{
A note on the texts
}

All citations from Conrad's works, with the exception of The Secret Agent which appeared as the first volume of the Cambridge Edition of the Works of Joseph Conrad (Cambridge University Press, 1990-), are from the Dent Collected Edition (1946-55). 